\title{
Two Separable Conformers of TATP and Analogs Exist at Room Temperature
}

\author{
C. Denekamp, L. Gottlieb, T. Tamiri, A. Tsoglin, R. Shilav and M. Kapon
}

Supporting information

Table of content:

Cartesian coordinates for optimized 1-D 3 S2

Cartesian coordinates for optimized $\mathbf{1 - \mathbf { C } _ { \mathbf { 2 } }} \quad \mathrm{S} 3$

Cartesian coordinates for optimized TS1

Cartesian coordinates for optimized 1- $\mathbf{C}_{2}-\mathbf{2} \mathbf{H}_{2} \mathbf{O}$ (diequatorial)

Cartesian coordinates for optimized 1- $\mathbf{C}_{2}-\mathbf{2} \mathbf{H}_{2} \mathbf{O}$ (diaxial) $\quad$ S6

Cartesian coordinates for optimized $\mathbf{T S 1}-\mathbf{2} \mathbf{H}_{\mathbf{2}} \mathbf{O} \quad$ S7

Cartesian coordinates for optimized $\mathbf{C}_{2}-\mathrm{H}_{3} \mathbf{O}^{+} \quad \mathrm{S} 8$

Cartesian coordinates for optimized 2- $\mathbf{C}_{\mathbf{3}} \quad$ S9

Cartesian coordinates for optimized 2-pseudo $\mathbf{C}_{3} \quad$ S10

Cartesian coordinates for optimized 2-pseudo $\mathbf{C}_{2} \quad$ S11 
1-D : $^{2}$

C, $0,-0.9602746278,0.2330967764,-1.7115444462$

$\mathrm{C}, 0,-0.9602746246,0.2330967761,1.7115444481$

$\mathrm{O}, 0,1.2859787392,0.232350801,-1.0609144799$

$\mathrm{O}, 0,0.1874518311,-0.5900108983,-1.5652529179$

$\mathrm{O}, 0,0.4370903472,0.4384097163,1.5652529173$

C, $0,1.9205492524,-0.4661935525,-0.0000000018$

$\mathrm{O}, 0,-1.5982498288,-0.1565502099,0.5043384404$

$\mathrm{O}, 0,-1.3486113166,0.8718704051,-0.5043384376$

$\mathrm{O}, 0,1.036340228,-0.7960698141,1.0609144777$

C, $0,-1.4542788232,1.5896464429,2.219947147$

$\mathrm{H}, 0,-2.5393611156,1.5473955292,2.3490860742$

$\mathrm{H}, 0,-0.9886853417,1.8285669441,3.1799769758$

$\mathrm{H}, 0,-1.2115734396,2.3726792699,1.4994032128$

C, $0,-0.7159212586,1.4104177474,-2.6586174177$

$\mathrm{H}, 0,-0.3871568581,1.0249701091,-3.6277457223$

$\mathrm{H}, 0,-1.6356519769,1.9856114313,-2.7956035475$

$\mathrm{H}, 0,0.0586285012,2.0643508293,-2.254050901$

C, $0,3.0206318655,0.5034079573,0.4386702702$

$\mathrm{H}, 0,3.5667593065,0.0651976798,1.2786596478$

$\mathrm{H}, 0,3.7167965799,0.6863596661,-0.3843734289$

$\mathrm{H}, 0,2.5823821712,1.4517356169,0.7546476874$

C, $0,-1.2828757762,-0.9252303516,2.6586174196$

$\mathrm{H}, 0,-0.8139844066,-0.733405437,3.6277457235$

$\mathrm{H}, 0,-2.3639581459,-1.0147472635,2.7956035513$

H,0,-0.8943296498,-1.8614929818,2.2540509017

C, $0,-2.0212333498,-0.7460016551,-2.2199471437$

$\mathrm{H}, 0,-2.9661886752,-0.2109800157,-2.3490860689$

$\mathrm{H}, 0,-1.7169915218,-1.1717917496,-3.1799769732$

$\mathrm{H}, 0,-2.1645315976,-1.5531645405,-1.4994032095$

C, $0,2.4536773422,-1.832240141,-0.4386702755$

$\mathrm{H}, 0,3.139931749,-1.6931778654,-1.2786596542$

$\mathrm{H}, 0,2.9884904064,-2.3139990283,0.3843734225$

H, $0,1.6294240146,-2.4741081937,-0.7546476915$ 
1-C : $^{2}$

C, $0,0.6056351127,-1.0430903497,1.5639409855$

C, $0,0.6155572128,-1.0142809542,3.097572048$ $\mathrm{H}, 0,1.6455943247,-1.0453226023,3.4608641805$ $\mathrm{H}, 0,0.1379092362,-0.0997480462,3.4596755034$ $\mathrm{H}, 0,0.0676387538,-1.8776783917,3.482171318$ C, $0,1.2042363874,-2.3191697707,0.9830200432$ $\mathrm{H}, 0,0.6068944916,-3.1812369546,1.2928872986$ $\mathrm{H}, 0,1.2255710593,-2.2760810131,-0.1044943811$ $\mathrm{H}, 0,2.2257772972,-2.4362086203,1.3560663493$ $\mathrm{O}, 0,1.3743462674,0.1126249578,1.234972484$ $\mathrm{O}, 0,-0.7752244461,-0.8814072648,1.256113169$ $\mathrm{O}, 0,1.6297070719,0.1546394262,-0.2036111816$ $\mathrm{O}, 0,-1.0692069334,-1.342413595,-0.101952317$ C, $0,1.1518345461,1.3887606333,-0.7207654109$ C, $0,1.6780753373,1.3881452184,-2.1574865733$ $\mathrm{H}, 0,1.2635164679,2.2488222523,-2.6893148708$ $\mathrm{H}, 0,2.7691404205,1.4568961603,-2.1558050339$ $\mathrm{H}, 0,1.3769032623,0.4757297186,-2.6740378419$ C, $0,1.6269288371,2.5966802982,0.0893660261$ $\mathrm{H}, 0,1.3603242827,3.5219769214,-0.4282252277$ $\mathrm{H}, 0,1.1703287938,2.5954540858,1.0800964501$ $\mathrm{H}, 0,2.7134445504,2.546177968,0.2007144446$ C, $0,-1.7518583484,-0.3389047571,-0.8466868762$ C,0,-2.4049668616,-1.1432005048,-1.9778412802 $\mathrm{H}, 0,-3.1430972591,-1.8303851629,-1.5576899585$ $\mathrm{H}, 0,-2.8970730549,-0.4635784806,-2.6777459937$ $\mathrm{H}, 0,-1.6472668092,-1.7200761516,-2.515188524$ $\mathrm{O}, 0,-0.2635329843,1.4925492238,-0.6512781174$ $\mathrm{O}, 0,-0.8560483144,0.5122840587,-1.5592996526$ C, $0,-2.7556566019,0.4486308753,-0.0122121852$ $\mathrm{H}, 0,-2.24712665,1.0443970062,0.7437371765$ $\mathrm{H}, 0,-3.3224125635,1.1140014578,-0.6698421247$ $\mathrm{H}, 0,-3.4451056217,-0.2407787331,0.4831354976$ 
TS1:

C, $, 0,-0.1509620882,-0.8796311065,1.637135303$

C, $0,-0.2031984457,-1.0959820632,3.166023156$ $\mathrm{H}, 0,0.601953083,-1.7744006853,3.4586433876$ $\mathrm{H}, 0,-0.0722198254,-0.1418210189,3.6820856278$ $\mathrm{H}, 0,-1.1688911498,-1.5220895539,3.4464813173$ C, $0,-0.3484183921,-2.1817538436,0.8785927331$ $\mathrm{H}, 0,-1.3624590853,-2.5493954754,1.0584649689$ $\mathrm{H}, 0,-0.1789872038,-2.0516907558,-0.1869725618$ $\mathrm{H}, 0,0.3656233955,-2.9225544305,1.25004381$ $\mathrm{O}, 0,1.1593829651,-0.3170799065,1.4734520065$ $\mathrm{O}, 0,-1.1736652349,0.1098334947,1.5233376408$ $\mathrm{O}, 0,1.744184496,-0.6007243372,0.1720263791$ $\mathrm{O}, 0,-1.9491810395,0.544731524,0.3251348532$ C, $0,1.816486675,0.5871876913,-0.5998290605$ C, $0,2.5017561834,0.123885363,-1.8882994731$ $\mathrm{H}, 0,2.5827563614,0.972921592,-2.572844991$ $\mathrm{H}, 0,3.5033835232,-0.2563823244,-1.6705544783$ $\mathrm{H}, 0,1.9187944491,-0.6642871955,-2.3689415601$ C, $0,2.5504605748,1.7236209002,0.1096888387$ $\mathrm{H}, 0,2.5942557256,2.6065553461,-0.5340394306$ $\mathrm{H}, 0,2.0349168325,1.977813853,1.0366762087$ $\mathrm{H}, 0,3.5693897004,1.4002632813,0.3404402021$ C, $0,-1.6387016604,0.3031192932,-1.062750127$ C, $0,-2.4020672941,-0.9121226789,-1.6067084127$ $\mathrm{H}, 0,-3.4711919262,-0.7418088528,-1.4541581119$ $\mathrm{H}, 0,-2.2137391324,-1.0358906041,-2.6775530686$ $\mathrm{H}, 0,-2.1210265905,-1.8301437371,-1.0908152128$ $\mathrm{O}, 0,0.5250906482,1.129437194,-0.8441759958$ $\mathrm{O}, 0,-0.2965253204,0.0227897252,-1.3524629966$ C, $0,-2.1176173323,1.6081454977,-1.7136474469$ $\mathrm{H}, 0,-1.4958381405,2.4387186735,-1.3778005341$ $\mathrm{H}, 0,-2.0514647704,1.5157849109,-2.8026311944$ 
1- $\mathbf{C}_{\mathbf{2}}-\mathbf{2} \mathbf{H}_{2} \mathbf{O}$ (diequatorial):

C, $0,1.049894785,-0.5204387786,1.3489046737$

C, $0,1.0478099225,-0.5092978443,2.8829118666$

$\mathrm{H}, 0,2.0777519624,-0.5241108567,3.247132366$

$\mathrm{H}, 0,0.556037011,0.3955008781,3.2507908736$

$\mathrm{H}, 0,0.5112837814,-1.3823007202,3.2616334997$

C, $0,1.7612704231,-1.7266659706,0.7492843834$ $\mathrm{H}, 0,1.2736713399,-2.6489972319,1.0782590611$

$\mathrm{H}, 0,1.7496994188,-1.6861238797,-0.3384772915$ $\mathrm{H}, 0,2.7985840214,-1.7320730636,1.0949494394$ $\mathrm{O}, 0,1.6769254612,0.7062032241,1.030370477$ $\mathrm{O}, 0,-0.3593119851,-0.4963977906,1.0317110298$ $\mathrm{O}, 0,1.9410540538,0.7820992756,-0.4062821335$ $\mathrm{O}, 0,-0.6154353619,-0.9462909762,-0.3407284386$ C, $0,1.3622483464,1.979453701,-0.9108789814$ C, $0,1.8841946505,2.0353457842,-2.347812694$ $\mathrm{H}, 0,1.3929113539,2.8585560521,-2.8733283974$ $\mathrm{H}, 0,2.9641183062,2.2038667341,-2.3448630023$ $\mathrm{H}, 0,1.6683488675,1.1033436085,-2.872086346$ C, $0,1.7362119872,3.2145715691,-0.0895387648$ $\mathrm{H}, 0,1.3987916516,4.1187872981,-0.602627514$ $\mathrm{H}, 0,1.2777668366,3.1718768317,0.8994815464$ $\mathrm{H}, 0,2.8229474999,3.2501774516,0.0250528116$ C, $0,-1.3925948682,0.0235895881,-1.0769292387$ C, $0,-1.9782827574,-0.8131256496,-2.2211425442$ $\mathrm{H}, 0,-2.6201802393,-1.5991032963,-1.8163848406$ $\mathrm{H}, 0,-2.5602629176,-0.1655884041,-2.881046556$ $\mathrm{H}, 0,-1.17285211,-1.2744755788,-2.7996316836$ $\mathrm{O}, 0,-0.0567000333,1.9585980956,-0.8332334756$ $\mathrm{O}, 0,-0.5625141712,0.945050364,-1.7585781884$ C, $0,-2.4521874005,0.695916594,-0.2136276336$ $\mathrm{H}, 0,-1.9954790148,1.3127651835,0.5584892949$ $\mathrm{H}, 0,-3.0774023463,1.327370415,-0.8507077442$ $\mathrm{H}, 0,-3.0806908902,-0.0642749408,0.2590072594$ $\mathrm{H}, 0,-1.6876050099,-2.7343132384,-0.2407203556$ $\mathrm{H}, 0,-1.4333766911,-1.7355108854,2.1418549195$ $\mathrm{O}, 0,-2.3213339514,-3.4480593367,-0.054433168$ $\mathrm{H}, 0,-2.753634526,-3.1399120184,0.7609480217$ $\mathrm{O}, 0,-1.8094593697,-2.5103103132,2.599757537$ $\mathrm{H}, 0,-1.3676159771,-3.258694639,2.1665791196$ 
$\mathbf{1 - C _ { 2 }}-\mathbf{- 2} \mathbf{H}_{2} \mathrm{O}$ (diaxial):

$\mathrm{C}, 0,0.6257630357,-0.5520587966,1.7202658758$

C, $0,0.2045413089,-0.2129869218,3.1559451522$

H,0,1.0942316313,-0.009312618,3.7567635149

$\mathrm{H}, 0,-0.4414673729,0.6690210535,3.1629131963$

$\mathrm{H}, 0,-0.3354693311,-1.0600689801,3.5860534014$

C, $0,1.4815317347,-1.8076160069,1.6132227355$

$\mathrm{H}, 0,0.9106959865,-2.6757420736,1.9547951527$

$\mathrm{H}, 0,1.8023081559,-1.9755122528,0.5863167908$

$\mathrm{H}, 0,2.3642023314,-1.6890940124,2.2477980321$

$\mathrm{O}, 0,1.3270615257,0.6224500122,1.321460832$

$\mathrm{O}, 0,-0.6308886441,-0.6678971343,1.0445736125$

$\mathrm{O}, 0,1.9320407321,0.4265988013,0.0037677816$

$\mathrm{O}, 0,-0.4853694371,-1.4158106411,-0.2133115658$

C, $0,1.4787315956,1.4629849808,-0.8544833778$

C, $0,2.3174898142,1.2666771754,-2.1179567318$

$\mathrm{H}, 0,1.942217924,1.9294829979,-2.902235097$

$\mathrm{H}, 0,3.3611859776,1.5142137181,-1.9078644788$

$\mathrm{H}, 0,2.2560316363,0.2354811768,-2.4671512826$

C, $0,1.6432424874,2.8598532789,-0.2489549751$

$\mathrm{H}, 0,1.4473547616,3.6178819105,-1.012439229$

$\mathrm{H}, 0,0.9633349245,3.0123915166,0.5908280295$

$\mathrm{H}, 0,2.6715910206,2.9690231664,0.1059308205$

C, $0,-1.0875832153,-0.7080047732,-1.3076473024$

C,0,-1.2461772982,-1.8041447061,-2.3698002878

$\mathrm{H}, 0,-1.6162862249,-1.3594169779,-3.2967299978$

$\mathrm{H}, 0,-0.2833858846,-2.2840372515,-2.5651688192$

$\mathrm{H}, 0,-1.9601296564,-2.5492380011,-2.0118042152$

$\mathrm{O}, 0,0.0736082831,1.3650009618,-1.0967878681$

$\mathrm{O}, 0,-0.1690560108,0.1832110363,-1.9268939858$

C, $0,-2.4158375724,-0.0568635156,-0.9384629875$

$\mathrm{H}, 0,-2.30294774,0.7245212028,-0.1880485216$

$\mathrm{H}, 0,-2.8570195523,0.3748922935,-1.8414734025$

$\mathrm{H}, 0,-3.0840304503,-0.8285001408,-0.5471348266$

$\mathrm{H}, 0,-0.8615914728,1.7535743332,0.9576390335$

$\mathrm{H}, 0,-1.8487894554,-2.7945370324,0.3397537204$

O,0,-1.3997122073,2.4225344562,1.4124960747

$\mathrm{H}, 0,-1.6279951425,3.0414148364,0.7025991486$

$\mathrm{O}, 0,-2.707357921,-3.2474847193,0.4129271763$

H, $0,-3.1268639756,-2.8123053386,1.1700339645$ 
TS1-2H 20 :

C, $0,0.4558307151,-0.3306901902,1.5711936832$

C, $0,0.4080619672,-0.3282831978,3.1145412392$

$\mathrm{H}, 0,1.4295491689,-0.3717402789,3.5002799962$

$\mathrm{H}, 0,-0.0703575049,0.5860029406,3.4750601349$

$\mathrm{H}, 0,-0.15959566,-1.197840465,3.4515500562$

C, $0,1.0994265699,-1.5966712659,1.0340879527$

$\mathrm{H}, 0,0.484669379,-2.4539627237,1.3199852279$

$\mathrm{H}, 0,1.2258023706,-1.553562072,-0.0444431615$

H, $0,2.089329244,-1.7043559626,1.4873989058$

$\mathrm{O}, 0,1.1863928977,0.8625222196,1.2889183404$

$\mathrm{O}, 0,-0.9540229638,-0.1481718281,1.3177251102$

$\mathrm{O}, 0,1.9125188561,0.7987380192,0.0290489653$

$\mathrm{O}, 0,-1.7589322796,-0.4424766957,0.0881538105$

C, $0,1.3268361701,1.6769764996,-0.9182041619$

C, $0,2.2414042436,1.531969885,-2.1373633953$

$\mathrm{H}, 0,1.8567717171,2.1590197588,-2.9465532567$

$\mathrm{H}, 0,3.2574444632,1.8506743476,-1.8900785133$

$\mathrm{H}, 0,2.26609466,0.4936271232,-2.4738625601$

C, $0,1.2010730995,3.1129627517,-0.4123648826$

$\mathrm{H}, 0,0.7657717339,3.7510257565,-1.1864215071$

$\mathrm{H}, 0,0.5689407298,3.1408235639,0.476157891$

$\mathrm{H}, 0,2.1962513671,3.4891076114,-0.1590835797$

C, $0,-1.2636709839,-0.6504636661,-1.2578873627$

C, $0,-1.1362982308,-2.1450614965,-1.5738680656$

$\mathrm{H}, 0,-2.1114852709,-2.6138075443,-1.4187876177$

$\mathrm{H}, 0,-0.8336408469,-2.2910511036,-2.6151968981$

$\mathrm{H}, 0,-0.4147579963,-2.6348854138,-0.9207023214$

$\mathrm{O}, 0,-0.0165560926,1.3106957934,-1.2091376736$

$\mathrm{O}, 0,0.0059941434,-0.1259358035,-1.5192545856$

C, $0,-2.362322876,0.0195650582,-2.093476623$

$\mathrm{H}, 0,-2.3703581003,1.0933632118,-1.9037312821$

$\mathrm{H}, 0,-2.170145525,-0.16517903,-3.1553767458$

$\mathrm{H}, 0,-3.3395335275,-0.3956027083,-1.8331387679$

$\mathrm{H}, 0,-1.8832685951,-1.9130779887,1.8442149678$

$\mathrm{O}, 0,-1.8848385715,-2.7925771258,2.2616512937$

$\mathrm{H}, 0,-2.5339737737,-2.7127619239,2.9759366405$ 


\section{$\mathrm{C}_{2}-\mathrm{H}_{3} \mathrm{O}^{+}$:}

C,0,1.4038028888,-0.8259814934,1.391494709

C, $0,1.2524951706,-1.0392618739,2.8583361893$ $\mathrm{H}, 0,2.2253581608,-1.3737774784,3.2464875902$ $\mathrm{H}, 0,0.9668851685,-0.1187824739,3.370877749$ $\mathrm{H}, 0,0.523761332,-1.8308650909,3.0492457928$ C, $0,1.6397145889,-1.9247115966,0.4291333469$ $\mathrm{H}, 0,1.4322650267,-2.8879032308,0.8960508387$ H, $0,1.0090627722,-1.7850366176,-0.4543306892$ $\mathrm{H}, 0,2.6864634548,-1.887733579,0.1009652117$ $\mathrm{O}, 0,1.6096086454,0.4069217307,1.0891702319$ $\mathrm{O}, 0,-0.9123966491,-0.7484536656,1.0042609904$ $\mathrm{O}, 0,1.939952765,0.5957060912,-0.323553891$ $\mathrm{O}, 0,-1.2326645401,-1.2630191757,-0.3156226285$ C, $0,1.2093561704,1.7730996058,-0.8100597483$ C, $0,1.755751022,1.8830515582,-2.2340034526$ $\mathrm{H}, 0,1.1864961877,2.6600072272,-2.7513838133$ $\mathrm{H}, 0,2.8099106898,2.1681442958,-2.2114290488$ $\mathrm{H}, 0,1.6419019038,0.9451114516,-2.7785167287$ C, $0,1.4758608307,3.0045430414,0.05056057$ $\mathrm{H}, 0,1.0616314705,3.8861369219,-0.4438237678$ $\mathrm{H}, 0,1.012134338,2.908177492,1.0344946039$ $\mathrm{H}, 0,2.5539889833,3.1373495983,0.1699844463$ C, $0,-1.6684595471,-0.202078807,-1.1911025994$ C, $0,-2.1491206221,-0.9733981831,-2.4248383456$ $\mathrm{H}, 0,-2.9769866335,-1.6297658203,-2.1471722226$ $\mathrm{H}, 0,-2.4910513294,-0.2655444456,-3.1829420012$ $\mathrm{H}, 0,-1.3389623052,-1.5769385517,-2.8423454393$ $\mathrm{O}, 0,-0.1667162914,1.5632625773,-0.7149095223$ $\mathrm{O}, 0,-0.5561613154,0.5285499018,-1.6887865606$ C, $0,-2.7404880657,0.6743747377,-0.56097729$ $\mathrm{H}, 0,-2.3724667492,1.1755315123,0.3330933789$ $\mathrm{H}, 0,-3.0594866519,1.4294791326,-1.2837784817$ $\mathrm{H}, 0,-3.6025712919,0.0555131869,-0.2967424791$ $\mathrm{H}, 0,-1.4096205204,-1.401717954,1.5695426335$ $\mathrm{O}, 0,-1.9390514341,-2.5918184953,2.6891694275$ $\mathrm{H}, 0,-2.2677782842,-3.4209948205,2.3061060374$ $\mathrm{H}, 0,-2.6049797845,-2.3334044054,3.3465317348$ 
2-C $\mathbf{C}_{3}$ :

$\mathrm{C}, 0,-1.1820787412,0.3510462195,-1.8441670958$

C, $0,-1.1708500396,0.3796582429,1.574015838$

$\mathrm{O}, 0,1.0734600025,0.2806250422,-1.2123430171$

$\mathrm{O}, 0,-0.0650988619,-0.51007963,-1.6866427331$

O,0,0.2296899294,0.5482431967,1.4092009436

C, $0,1.6912366826,-0.416303588,-0.1439582866$

$\mathrm{O}, 0,-1.8262338239,-0.0044518049,0.3788018012$

$\mathrm{O}, 0,-1.5522251659,1.0087682294,-0.6446157952$

$\mathrm{O}, 0,0.7964903536,-0.709059038,0.917912612$

C,0,-1.6323496606,1.7530274673,2.09447789

$\mathrm{H}, 0,-2.7289646748,1.7357575265,2.0732735266$

$\mathrm{H}, 0,-1.3037340674,2.5257321793,1.3942081601$

C, $0,-0.9051141137,1.5152690788,-2.8111053337$

$\mathrm{H}, 0,-1.7948176051,2.1563135243,-2.8248198281$

$\mathrm{H}, 0,-0.077161497,2.1093757671,-2.4159775104$

C, $0,2.8299946469,0.5271094207,0.2800436776$

$\mathrm{H}, 0,3.4309062436,0.7465439054,-0.6107227765$

$\mathrm{H}, 0,2.3955213169,1.4692116671,0.6241819689$

C, $0,-1.5216106611,-0.7546879522,2.5523854617$

$\mathrm{H}, 0,-2.6138105472,-0.8515655648,2.5726331863$

$\mathrm{H}, 0,-1.1153964354,-1.6920552295,2.1635629679$

C, $0,-2.2797231807,-0.5902042142,-2.373426842$

$\mathrm{H}, 0,-3.2154739837,-0.0185753134,-2.3416401161$

$\mathrm{H}, 0,-2.3874343584,-1.4318706581,-1.683594692$

C,0,2.2148517089,-1.803404104,-0.558133571

H,0,2.7718052551,-1.6549414258,-1.4915241276

$\mathrm{H}, 0,1.3626488017,-2.4501023695,-0.7846825797$

C, $0,-0.5998599735,1.0023129747,-4.2262748164$

$\mathrm{H}, 0,-0.4463109003,1.8586366052,-4.894415019$

$\mathrm{H}, 0,0.3419928492,0.4399149974,-4.2071262447$

C,0,-1.996687831,-1.0721086804,-3.8056516603

$\mathrm{H}, 0,-2.8486819541,-1.6639527041,-4.16308569$

H,0,-1.1276615034,-1.7413500029,-3.7958502495

C, $0,-1.7262351644,0.1028923138,-4.7569288222$

$\mathrm{H}, 0,-2.6442786736,0.699311692,-4.8689363747$

$\mathrm{H}, 0,-1.4728945148,-0.2718520957,-5.7566359785$

C, $0,3.7065216839,-0.1140859773,1.3663460363$

$\mathrm{H}, 0,4.5283119102,0.5685773863,1.6149466507$

$\mathrm{H}, 0,3.1097436419,-0.2394743965,2.2785741545$

C,0,3.1247853768,-2.4299122745,0.5108798323

$\mathrm{H}, 0,3.5380977376,-3.3712134598,0.1274958893$

$\mathrm{H}, 0,2.5242761839,-2.6840199521,1.3930368615$

C,0,4.2574607067,-1.47653094,0.9202175344

$\mathrm{H}, 0,4.9371167377,-1.3310552586,0.0671197447$

$\mathrm{H}, 0,4.8557353076,-1.924434074,1.723584802$

C, $0,-1.1316574866,2.0374047705,3.5195891037$

$\mathrm{H}, 0,-1.5590582301,2.9846776291,3.8714977029$

$\mathrm{H}, 0,-0.0427682542,2.1692149266,3.5000534629$

C, $0,-0.9900076558,-0.4566425404,3.9626522707$

H,0,-1.2941 129424,-1.2641120788,4.6399497138

$\mathrm{H}, 0,0.1069080739,-0.457465481,3.938813565$

C, $0,-1.4921091305,0.8979880203,4.4843319927$

$\mathrm{H}, 0,-2.5848885506,0.8565760521,4.6071831324$

$\mathrm{H}, 0,-1.0736778396,1.0988588178,5.4786259502$ 
2-pseudo $\mathrm{C}_{3}$ :

C, $0,-0.8941810929,0.3644341972,-1.7318715017$

$\mathrm{C}, 0,-0.9605061813,0.3803006996,1.6924982313$

$\mathrm{O}, 0,1.3432306826,0.3691166868,-1.0392692033$

$\mathrm{O}, 0,0.246970591,-0.4575302954,-1.550697802$

$\mathrm{O}, 0,0.4380658403,0.5856531405,1.5766118706$

C, $0,1.9568671992,-0.3176027704,0.0393732703$

$\mathrm{O}, 0,-1.5648146435,-0.0241091875,0.4751483933$

$\mathrm{O}, 0,-1.3126046371,1.0044541781,-0.5373426678$

$\mathrm{O}, 0,1.0473678913,-0.6490039185,1.0755494089$

C, $0,-1.4774094888,1.7413337431,2.1909254715$

$\mathrm{H}, 0,-1.252594343,2.4994429385,1.4363351508$

C, $0,-0.6355551857,1.5399426402,-2.6906844287$

$\mathrm{H}, 0,-1.5456928509,2.1509361838,-2.7240389899$

$\mathrm{H}, 0,0.1631632947,2.1607608292,-2.276786471$

C, $0,3.0551041066,0.6570512214,0.4997589563$

H, $0,3.6717455114,0.9028037513,-0.3732446398$

$\mathrm{H}, 0,2.5826995781,1.5819515564,0.8404513187$

C, $0,-1.3170697735,-0.7618499289,2.6610318963$

$\mathrm{H}, 0,-0.9852028603,-1.7096163409,2.228128637$

C, $0,-1.9482665896,-0.610852992,-2.2863277923$

$\mathrm{H}, 0,-2.9025499756,-0.069840363,-2.2767528994$

$\mathrm{H}, 0,-2.0443193007,-1.4562510909,-1.5993940381$

C, $0,2.5342967017,-1.6831862399,-0.3747041033$

$\mathrm{H}, 0,3.1095164779,-1.5080662909,-1.2922542853$

H, $0,1.7090094923,-2.3541345104,-0.6287345975$

C, $0,-0.2813087379,1.0386881426,-4.0988038877$

$\mathrm{H}, 0,-0.1413702659,1.9003730053,-4.7629854102$

$\mathrm{H}, 0,0.6782834891,0.5085699377,-4.0577380089$

C, $0,-1.6170982248,-1.0822791152,-3.7115741128$

$\mathrm{H}, 0,-2.440849426,-1.7011976649,-4.0888187164$

$\mathrm{H}, 0,-0.7272183189,-1.7229592748,-3.6820693917$

C, $0,-1.3635955939,0.1022446113,-4.6559687967$

$\mathrm{H}, 0,-2.2980362908,0.6676853947,-4.7902284918$

$\mathrm{H}, 0,-1.0736595737,-0.2627822026,-5.6493281502$

C,0,3.9226157705,0.033011764,1.6031833064

$\mathrm{H}, 0,4.7158880106,0.7384040156,1.8795193297$

$\mathrm{H}, 0,3.3062175998,-0.1200929347,2.4976894306$

C, $0,3.4360229396,-2.2911030572,0.7118358569$

$\mathrm{H}, 0,3.8886843883,-3.2153691579,0.3313499943$

$\mathrm{H}, 0,2.8210321858,-2.5717832036,1.5756835695$

C, $0,4.5273214734,-1.3069995685,1.1588808152$

$\mathrm{H}, 0,5.2236683893,-1.1316810579,0.3250386515$

$\mathrm{H}, 0,5.1186678632,-1.7441307427,1.9733107138$

$\mathrm{H}, 0,-0.7257208919,-0.5905884361,3.5690071852$

$\mathrm{H}, 0,-0.9169545323,2.0005273768,3.0972836285$

C, $0,-2.9819041907,1.6847332988,2.4956205806$

H, $0,-3.3074594683,2.6577750779,2.8834660792$

$\mathrm{H}, 0,-3.529126039,1.5150800168,1.5600485778$

C, $0,-2.8162004542,-0.7954260107,2.999738257$

$\mathrm{H}, 0,-2.9990153465,-1.5676714112,3.7574687237$

$\mathrm{H}, 0,-3.3814898949,-1.087995866,2.1063858235$

$\mathrm{C}, 0,-3.3157543063,0.5692463168,3.4970879816$

$\mathrm{H}, 0,-4.3977423023,0.5324155914,3.6760174367$

$\mathrm{H}, 0,-2.8455706246,0.7986683262,4.4651898401$ 
2-pseudo $\mathrm{C}_{2}$

C, $0,-0.9662466318,0.2831352528,-1.6725569728$

C, $0,-0.8613508891,0.2919779899,1.7707463811$

$\mathrm{O}, 0,1.2966824076,0.4092876463,-0.9739096056$

$\mathrm{O}, 0,0.3118625745,-0.3397766356,-1.7578103705$

$\mathrm{O}, 0,0.5572689117,0.1774533739,1.7816841542$

C, $0,1.9015963947,-0.4680203329,-0.0372716641$

$\mathrm{O}, 0,-1.3378174439,0.9288644502,0.5906254014$

$\mathrm{O}, 0,-1.6401634865,-0.0464133787,-0.4640702033$

$\mathrm{O}, 0,0.9763235347,-0.9389112351,0.9303379988$

C, $0,-1.1234260349,1.3493739126,2.8648441442$

$\mathrm{H}, 0,-0.6607493132,2.2931019454,2.5562032929$

C, $0,-0.9125144611,1.7894292515,-1.9479140547$

$\mathrm{H}, 0,-1.9286936867,2.1795287229,-1.8119325543$

$\mathrm{H}, 0,-0.2777862512,2.270227697,-1.2035175855$

C, $0,3.0336541627,0.3819322508,0.5644001856$

$\mathrm{H}, 0,3.6567707301,0.7317701586,-0.2671000176$

$\mathrm{H}, 0,2.5998074973,1.2614410284,1.0451870175$

C, $0,-1.5630355686,-1.0345897842,2.0798400497$

$\mathrm{H}, 0,-1.3951309386,-1.7333698498,1.2602348781$

C, $0,-1.7808240601,-0.4697706903,-2.7472429568$

$\mathrm{H}, 0,-2.824855154,-0.1599750393,-2.625811069$

$\mathrm{H}, 0,-1.7311101078,-1.5436162627,-2.5368087268$

C, $0,2.437114994,-1.762772559,-0.675058255$

$\mathrm{H}, 0,3.0102839815,-1.4595409608,-1.5598489202$

$\mathrm{H}, 0,1.5932735702,-2.362794966,-1.0246829313$

C, $0,-0.4138204015,2.0825909017,-3.3712172747$

$\mathrm{H}, 0,-0.438167908,3.165651242,-3.5437505239$

$\mathrm{H}, 0,0.635000143,1.7717734213,-3.454211156$

C, $0,-1.2913820589,-0.1569803619,-4.1696784757$

$\mathrm{H}, 0,-1.9506452785,-0.6552917486,-4.8913800567$

$\mathrm{H}, 0,-0.28911799,-0.5795908592,-4.306979036$

$\mathrm{C}, 0,-1.2523075861,1.355370869,-4.4324235247$

$\mathrm{H}, 0,-2.2781175579,1.7535611226,-4.4210775906$

$\mathrm{H}, 0,-0.8516750872,1.552373482,-5.4347106559$

C, $0,3.879821208,-0.4349353473,1.5517363849$

$\mathrm{H}, 0,4.694029696,0.1939363863,1.9323251534$

$\mathrm{H}, 0,3.2598446108,-0.7102513008,2.4137933733$

C, $0,3.3232816263,-2.5637679203,0.2924992791$

$\mathrm{H}, 0,3.7475656343,-3.4254126664,-0.2380674783$

$\mathrm{H}, 0,2.6995858759,-2.9647295215,1.1006506599$

C, $0,4.4440912749,-1.7019798964,0.8923608834$

$\mathrm{H}, 0,5.147736185,-1.4131190459,0.0971465026$

$\mathrm{H}, 0,5.019076369,-2.2860631047,1.6218395076$

$\mathrm{H}, 0,-1.0720959314,-1.4469363424,2.9700664719$

$\mathrm{H}, 0,-0.6058496126,1.0112569366,3.7697831698$

C, $0,-2.6242022477,1.5305862737,3.1381209479$

$\mathrm{H}, 0,-2.7529012557,2.236041598,3.968439318$

$\mathrm{H}, 0,-3.0949224794,1.9832345159,2.2574196631$

C, $0,-3.0630775195,-0.8371828935,2.3459641025$

$\mathrm{H}, 0,-3.5106042195,-1.8016224363,2.6163900613$

$\mathrm{H}, 0,-3.5524775923,-0.5087490753,1.4209507767$

C, $0,-3.3098138457,0.1940646724,3.4570963583$

$\mathrm{H}, 0,-4.3865412286,0.3494086273,3.6001577803$

$\mathrm{H}, 0,-2.9201348176,-0.1970470045,4.4089584462$ 